\title{
Afrontamiento, funcionamiento familiar y calidad de vida relacionada con la salud en cuidadores venezolanos de pacientes con cáncer
}

\author{
Coping, family functioning, and health-related quality of life \\ in Venezuelan cancer patients' caregivers
}

\author{
Neilú Hernández, Anthony Salas y José Javier Altuve ${ }^{l}$ \\ Citación: Hernández, N., Salas, A. y Altuve, J.J. (2020). Afrontamiento, funcionamiento familiar \\ y calidad de vida relacionada con la salud en cuidadores venezolanos de pacientes con cáncer. \\ Psicología y Salud, 30(2), 161-172. https://doi.org/10.25009/pys.v30i2.2651.
}

\section{RESUMEN}

\begin{abstract}
El presente estudio correlacional, de campo y diseño no experimental tuvo como objetivo determinar la correlación entre el afrontamiento, el funcionamiento familiar y la calidad de vida relacionada con la salud en una muestra de cuidadores de pacientes oncológicos venezolanos. Participaron 200 cuidadores de ambos sexos, con edades de 18 a 82 años, de pacientes oncológicos que asistían a dos centros especializados en oncología en la ciudad de Caracas, a quienes se aplicó el cuestionario SF-12, la Escala de Estilos de Afrontamiento y la escala FACES-III de funcionamiento familiar. Los resultados mostraron una correlación directa entre los estilos de afrontamiento racional y por desapego con la cohesión y adaptabilidad familiar. El funcionamiento familiar y el estilo de afrontamiento no correlacionaron con la calidad de vida relacionada con la salud de los cuidadores, excepto el estilo emocional, que tuvo una correlación inversa y significativa.
\end{abstract}

Palabras clave: Afrontamiento; Calidad de vida relacionada con la salud; Cuidadores; Funcionamiento familiar; Pacientes con cáncer.

\begin{abstract}
The present correlational, field-test, non-experimental study sought to determine the correlation between coping, family functioning and health-related quality of life of a sample of caregivers of Venezuelan cancer patients. Participants consisted of 200 caregivers of both sexes, aged 18 to 82 years attending two specialized oncology centers at the city of Caracas. Participants responded to the SF-12 questionnaire, the Coping Styles Scale, and the FACES-III Family Functioning Scale. Results showed a direct correlation between rational coping styles and detachment with family cohesion and adaptability. Family functioning and coping style did not correlate with quality of life related to the health of caregivers, except for the emotional style, that showed a significant and inverse correlation.
\end{abstract}

Key words: Coping; Health-related Quality of life; Caregivers; Family functioning; Cancer patients.

\footnotetext{
${ }^{1}$ Escuela de Psicología, Facultad de Humanidades y Educación, Universidad Central de Venezuela, Urbanización Valle Abajo, Parroquia San Pedro, Caracas, Venezuela, tel. (58)21-26-05-29-91, correos electrónicos: neilujose@gmail.com, a.salas360@gmail.com y a.altuve.07@gmail.com. Artículo recibido el 27 de agosto de 2019 y aceptado el 27 de enero de 2020.
} 
E cáncer es un conjunto de enfermedades por las que el organismo desarrolla células malignas que crecen de forma descontrolada (Velázquez, García, Alvarado y Sánchez, 2007). Esta enfermedad, de acuerdo con datos de la Organización Mundial de Salud (OMS) (2018), representa la segunda causa de muerte en el mundo.

El diagnóstico de cáncer no solo afecta al paciente, sino también a los miembros de su familia, sus relaciones y funcionamiento. Los familiares son los que frecuentemente deben asumir la responsabilidad del cuidado instrumental y emocional del paciente, así como su acompañamiento dentro y fuera de su domicilio; las personas que llevan a cabo tales funciones con el tiempo pasan a ser los cuidadores informales (Alderfer y Kazak, 2006; Garassini, 2016; Holland et al., 2015; Kissane y Parne, 2015). Un cuidador, de acuerdo con Hanly et al. (2016), es un miembro de la familia, un amigo $\mathrm{u}$ otra persona, quien provee ayuda en las tareas de cuidado al paciente desde su diagnóstico. Los cuidadores informales desempeñan actividades como acompañar al enfermo a las consultas médicas y sesiones de aplicación de los tratamientos, a la vez que se encargan de la compra de medicamentos, vigilan el régimen de la alimentación y brindan apoyo emocional al familiar diagnosticado (González, Fonseca, Valladares y López, 2017).

La sobrecarga en el cuidado del familiar diagnosticado incide de forma negativa tanto en la salud física como mental de todos los miembros de la familia, pero sobre todo en quienes asumen el rol de cuidadores primarios (Kissane y Parne, 2015; Garassini, 2016; Williams, 2018). Algunas consecuencias de la sobrecarga en la salud de los cuidadores de pacientes oncológicos son síntomas físicos tales como dolores musculares, fatiga, alteraciones del sueño o insomnio y respuesta inmunológica reducida. Los síntomas psicológicos que pueden manifestarse son ansiedad, incertidumbre, depresión, sensación de fracaso o desesperanza, dificultades cognitivas y comportamentales, tales como la falta de atención y concentración, una escasa capacidad de organización de las tareas y el aislamiento social. Otras consecuencias se encuentran asociadas a los gastos económicos y a la dificultad de acceder a otras oportunidades de empleo (Bayen, Laigle-Donadey y Proute, 2016; Ex- pósito, 2008; Lambert, Levesque y Girgis, 2016; Leroy, Fournier, Penel y Christophe, 2016; Rogero, 2010; Rumpold et al., 2016; Williams, 2018).

Así pues, el agotamiento o la sobrecarga de los cuidadores se vuelve un asunto de salud pública en sí mismo debido a todas las consecuencias que trae consigo (Ariza, Barrera y Pinto, 2009; García, 2011). La OMS (2018) define la salud como un estado de bienestar físico, mental, social y espiritual completo que no se adjudica a la ausencia de enfermedad. En la literatura especializada, a la salud también se le conoce con el nombre de calidad de vida relacionada a la salud, o CVRS, que comprende un nivel de bienestar que evalúa la persona en relación con diferentes dominios de su vida y el impacto que tiene sobre esta (Urzúa, 2010). Aunque ambos conceptos son difíciles de diferenciar (Karimi y Braizer, 2016), el concepto de "calidad de vida relacionada a la salud" se diferencia del de "calidad de vida" en cuanto que el primero tiene que ver con los aspectos de la vida relacionados únicamente con la salud, la enfermedad y los tratamientos (Fernández, Fernández y Cieza, 2010).

Si bien se ha reportado que hay variables sociodemográficas, de la propia enfermedad y psicológicas que pueden deteriorar la salud física y mental de los cuidadores (Lambert, Levesque y Girgis, 2016; Williams, 2018), también hay otras que incrementan la calidad de vida y la salud, tales como las estrategias de afrontamiento y el funcionamiento familiar adecuado.

Como se ha señalado antes, los cuidadores familiares que proveen cuidados a pacientes oncológicos tienen que afrontar una variedad de problemas físicos, sociales y económicos en el proceso de cuidar (Tokem, Hanife y Atiye, 2015). El afrontamiento es un concepto ampliamente conocido en la literatura sobre el estrés. Lazarus y Folkman (1986) lo han definido como aquellos esfuerzos cognitivos y conductuales constantemente cambiantes que se ponen en marcha para el manejo de las demandas internas o externas que se han interpretado como amenazantes y que desbordan los recursos del individuo. El afrontamiento del cáncer son las respuestas de los pacientes y sus cuidadores ante la enfermedad, las que se componen de la valoración y las reacciones consecuentes de ellos ante este estresor (Ariza et al., 2009; Watson 
y Greer, 1998). Los mismos Lazarus y Folkman (1986) establecieron que hay dos formas de afrontamiento: el dirigido al problema, que incluye estrategias hacia su solución a través de diferentes alternativas, y el dirigido a la emoción, que implica la regulación de la respuesta emocional ante el estresor a partir del autocontrol, el distanciamiento o la reevaluación positiva.

Roger, Jarvis y Najarian (1993) han propuesto un modelo parsimonioso sobre el afrontamiento, que entienden como la propensión a emplear estrategias específicas ante los estresores, o predisposiciones generales que presenta un individuo para hacer frente a una situación. Roger et al. (1993) estudiaron los estilos de afrontamiento como una diferencia individual que puede tener una influencia importante en la relación entre el estrés y la salud de las personas (Sojo, Bethelmhy y Guarino, 2007). Dichos autores identificaron cuatro estilos de afrontamiento, dos adaptativos y dos desadaptativos. Los estilos adaptativos son el estilo de afrontamiento racional, orientado a la solución de problemas, y el estilo por desapego emocional, que implica una tendencia a afrontar las situaciones sin involucrarse emocionalmente con ellas, evaluándolas desde afuera con una perspectiva más objetiva. Los estilos desadaptativos, a su vez, son el emocional, que implica un manejo a partir del desbalance emocional producto de la situación de estrés, y el evitativo, que es una tendencia a obviar la ocurrencia de la situación estresante. Así, los estilos adaptativos de afrontamiento se asocian a un incremento de la salud, mientras que los desadaptativos a su deterioro.

Algunos estudios (Sojo et al., 2007; Torres y Guarino, 2013) han aportado evidencias sobre las relaciones entre los distintos estilos de afrontamiento y la salud en diversas muestras. Aldana y Guarino (2012), reportaron una relación significativa y positiva entre los estilos de afrontamientos adaptativos y la salud de una muestra de cuidadores de pacientes con afección por demencia tipo alzheimer, y una relación negativa con los estilos desadaptativos, respectivamente. Por su parte, Pérez, Frías, Romero y Del Pino (2016) encontraron que aquellos cuidadores de pacientes oncológicos que emplean estrategias de afrontamiento enfocadas en la solución de los problemas pueden mitigar de forma efectiva el disturbio emocional y el agotamiento percibido. Aldana y Guarino (2012) reportaron a su vez un mayor empleo del afrontamiento racional y emocional en la muestra de cuidadores de pacientes con afección por demencia tipo alzheimer. Un estudio de Flórez y Klimenko (2011) con una muestra de cuidadores de pacientes oncológicos encontró que la estrategia de afrontamiento activo fue la más empleada, seguida por la de planeación o planificación.

Como se señaló líneas atrás, el cáncer en uno de sus miembros es un estresor sumamente potente para la familia. La forma en cómo sobrelleve la crisis de la enfermedad determinará el modo en que empleen sus recursos de afrontamiento, asuman los diferentes roles y el grado de éxito de los mismos. Los conflictos entre los miembros pueden afectar el curso de la enfermedad del paciente, así como sus lazos y unidad, en tanto que el apoyo mutuo y la confianza en la destreza con el que se lleva a cabo el cuidado ayudan a la protección ante el estrés crónico (Fuchs et al., 2013). Por consiguiente, el funcionamiento familiar tiene una influencia decisiva tanto en la salud de pacientes oncológicos como en la de sus cuidadores (Jung et al., 2017).

El funcionamiento familiar hace referencia a la interacción afectiva que existe entre los miembros familiares, la cual hace cambiar su estructura con la finalidad de que logren superar sus dificultades evolutivas (Alderfer y Stanley, 2012; Van Schoors, Caes, Verhofstadt, Goubert y Alderfer, 2015). En el modelo circumplejo desarrollado por Olson (2000) y Olson, Waldvogel y Schlieff (2019), los autores establecen el funcionamiento familiar a partir de dos dimensiones básicas: la cohesión y la adaptabilidad. La cohesión alude al nivel de apego o desapego de los miembros de la familia. En esta dimensión se consideran los lazos emocionales, los límites, la toma de decisiones y los intereses compartidos por la familia. La adaptabilidad, por su parte, se refiere al grado de flexibilidad y capacidad de cambio de los miembros de la familia. En esta dimensión se considera el cambio de roles, el liderazgo, los estilos de negociación y la retroalimentación entre aquellos (Olson, 2000; Olson et al., 2019).

Los estudios reportan que un funcionamiento familiar percibido como favorable se asocia con el empleo de estrategias de afrontamiento adapta- 
tivas en los pacientes oncológicos (Acosta, López, Martínez y Zapata, 2017; Biffi y Mamede, 2010; Díaz y Yaringaño, 2010; Ramírez, 2017). Un estudio en que se empleó una muestra de cuidadores familiares de pacientes oncológicos pediátricos fue el de Martin et al. (2012), quienes hallaron que un mejor funcionamiento familiar se vinculaba con el uso de estrategias de afrontamiento activo, tales como la reevaluación positiva. Por su parte, Cortés, Bueno, Narváez, García y Guerrero (2012) encontraron que los familiares con bajo funcionamiento familiar tendían a reportar un mayor distrés psicológico que aquellos que quienes reportaban una alta funcionalidad.

El estudio de la sobrecarga de los cuidadores de pacientes oncológicos y su influencia en su calidad de vida ha sido un tema de amplia atención en la psicooncología. Las investigaciones en el área han mostrado la importancia del afrontamiento y el funcionamiento familiar como factores protectores de la salud en pacientes con cáncer; sin embargo, muy pocas se han realizado sobre los cuidadores, por lo que se hace necesario seguir examinando los estilos de afrontamiento y el funcionamiento familiar percibido por ellos, y específicamente la relación que guardan con su calidad de vida relacionada con la salud, toda vez que la sobrecarga es un factor que la afecta negativamente.

Por lo tanto, el presente estudio correlacional y no experimental (Kerlinger y Lee, 2002; Hernández, Fernández y Baptista, 2012) tuvo como objetivo determinar la relación entre el afrontamiento, el funcionamiento familiar y la calidad de vida relacionada con la salud en una muestra de cuidadores de pacientes oncológicos venezolanos.

\section{MÉTODO}

\section{Participantes}

Participaron cuidadores de pacientes oncológicos que asistían a dos hospitales especializados en oncología: el Instituto Oncológico Luis Razzetti y el Servicio Oncológico Hospitalario del Instituto Venezolano de los Seguros Sociales (Antiguo Hospital Oncológico "Padre Machado"), ambos ubicados en la ciudad de Caracas (Venezuela). La muestra estuvo conformada por 200 cuidadores informales, de ambos sexos, con edades de entre 18 y 82 años. Específicamente, 85 pacientes (42.5\%) asistían al primero de ellos y $115(57.5 \%)$ al segundo. Los cuidadores encuestados en las áreas de consulta de ambos centros fueron 130 (65\%), y $70(35 \%)$ en las áreas de hospitalización. De los participantes, 155 fueron mujeres $(77.5 \%)$ y 45 hombres $(22.5 \%)$. La mayoría de los cuidadores eran hijos de los pacientes, casados, con instrucción universitaria, de estrato socioeconómico medio, con dedicación al tiempo de cuidado de entre 13 y 24 horas diarias ( $M=17$ horas). Respecto al familiar diagnosticado de los cuidadores, se reportó que en la mayor frecuencia correspondió a mujeres (122). De toda la muestra, 191 personas respondieron no tener antecedentes psiquiátricos $(95 \%)$ y $9(5 \%)$ sí lo reportaron. Por su parte, 126 $(63 \%)$ dijeron no tener antecedentes médicos, a diferencia de 74 personas (37\%) que sí los tenían. Los tipos de cáncer más frecuentes en los familiares de los cuidadores fueron mamarios (65 pacientes), ginecológicos (75 pacientes) y genitourinarios (41 pacientes), mientras que el resto lo desconocían (19 pacientes).

El muestreo realizado fue de tipo no probabilístico (Kerlinger y Lee, 2002), puesto que la elección de la muestra se hace en función de las características sociodemográficas de interés para la investigación. Los requisitos de inclusión que se tomaron en cuenta fueron que los participantes debían ser cuidadores de pacientes oncológicos que asistían a las instituciones ya señaladas, mayores de edad, parientes directos del paciente diagnosticado, que no tuvieran evidencia actual de trastornos psiquiátricos o emocionales significativos, y que supieran leer y escribir.

\section{Instrumentos}

\section{Cuestionario de Salud SF-12, versión 2 (Ware, Kosinski y Keller, 1996).}

Se empleó la versión adaptada al castellano de Ramírez, Agredo y Jerez (2010). Es esta una escala que evalúa la calidad de vida relacionada con la salud. Consta de doce ítems integrados en ocho dimensiones: función física (dos ítems), rol físico (dos), dolor corporal (uno), salud general (uno), 
vitalidad (uno), función social (uno), rol emocional (dos) y salud mental (dos ítems). Estas dimensiones se agrupan a su vez en dos componentes sumatorios: salud física y salud mental. El número de opciones de respuesta oscila entre tres y seis según el ítem; los puntajes mayores indican una alta calidad de vida relacionada con la salud. Los índices de confiabilidad reportados por Ramírez et al. (2010) fueron de entre 0.70 y 0.82 en las escalas de salud física y mental en ambos sexos.

Tres expertos en el área de la psicología de la salud y la psicooncología revisaron el cuestionario para su validación en la muestra de cuidadores. Una vez que se atendieron las consideraciones de los expertos, se hizo un estudio piloto que contó con la participación de 100 cuidadores de pacientes oncológicos de ambos sexos con edades de 18 a 82 años, estudio gracias al cual se logró obtener la versión definitiva que se administró a la muestra recabada. Los coeficientes de confiabilidad obtenidos fueron para las dimensiones de salud física, salud mental y salud total fueron aceptables: $0.72,0.73$ y 0.83 , respectivamente.

\section{Cuestionario de Estilos de Afrontamiento (CSQ) (Rosenstiel y Keefe (1983).}

Se empleó la versión adaptada al castellano y validada en Venezuela por Sojo et al. (2007). Mide los estilos de afrontamiento en un continuo de respuesta que va de 1 ("Nunca") a 4 ("Siempre"). Está conformada por cuatro dimensiones: afrontamiento emocional (doce ítems), desapego emocional (trece), afrontamiento racional (diez) y afrontamiento por evitación (cinco ítems), teniendo así un total de 40 ítems descriptivos de manifestaciones conductuales, cognitivas y emocionales en el afrontamiento. Puntajes mayores en una dimensión indican la presencia de un estilo de afrontamiento específico. La subescala de afrontamiento emocional presenta un coeficiente alfa de Cronbach de 0.77; la de desapego emocional, de 0.59; la de afrontamiento racional, de 0.68 , y la de afrontamiento evitativo, de 0.67 (Sojo et al., 2007).

\section{Escala de Adaptabilidad y Cohesión Familiar} (FACES III) (Olson, Portner y Bell, 1982).

Se utilizó la versión adaptada al castellano de Bazo et al. (2016), conformada por un total de 20 ítems que se responden según una escala tipo Likert que va de 1 a 5 ("Casi siempre", "Muchas veces", "A veces sí y a veces no", "Pocas veces", "Casi nunca") y que conforman las subescalas de Cohesión y Adaptabilidad, conformadas por diez ítems cada una. En la escala, los puntajes medios indican una mejor cohesión y adaptabilidad familiar. La escala ha reportado índices de confiabilidad aceptables, de entre 0.70 y 0.90 (Bazo et al., 2016). Los coeficientes de consistencia interna reportados en la muestra de cuidadores de pacientes oncológicos venezolanos fue 0.72 para la dimensión de cohesión familiar, y de 0.68 para adaptabilidad familiar, por lo que está dentro de rango aceptable de confiabilidad.

\section{Procedimiento}

En primer lugar, se redactaron sendas cartas a los respectivos directores de los centros especializados en oncología ya referidos, en las que se les solicitaba la aprobación para poder llevar a cabo la recolección de los datos del estudio. Para lo anterior, se diseñó una charla preventiva sobre el estrés para los cuidadores que estaban en la sala de consulta, y para los que estaban en el área de hospitalización se procedió a ir a las habitaciones, previa autorización y acompañamiento de los psicólogos de la unidad.

A cada cuidador se le pidió su colaboración, considerando antes su estado emocional y físico; si la respuesta era afirmativa, se les entregaba la carta de consentimiento informado en la que se consideraban aspectos relativos a la participación voluntaria, el uso y la confidencialidad de los resultados y el anonimato. La aplicación de los instrumentos se guío bajo los principios éticos para su validación y administración.

\section{Análisis de datos}

Los datos recolectados fueron procesados mediante el paquete estadístico SPSS, versión 22. Para el análisis se emplearon medidas de tendencia central y variabilidad. En el análisis de las correlaciones se empleó la prueba de momento de Pearson, y para el análisis de la consistencia interna el coeficiente alfa de Cronbach, para así dar cuenta de la confiabilidad de las escalas usadas en el estudio. 


\section{RESULTADOS}

\section{Análisis descriptivos de las variables}

En la Tabla 1 se resumen las medias y desviaciones típicas de las variables analizadas.

Tabla 1. Descriptivos de estilos de afrontamiento, funcionamiento familiar y salud.

\begin{tabular}{|l|r|c|c|c|}
\hline \multicolumn{1}{|c|}{ Estilo } & Mín. & Máx. & M & D.E. \\
\hline Afrontamiento emocional & 11 & 38 & 21.09 & 5.11 \\
\hline Afrontamiento racional & 16 & 44 & 33.43 & 5.38 \\
\hline Afrontamiento por desapego & 15 & 45 & 27.84 & 5.82 \\
\hline Afrontamiento evitativo & 7 & 25 & 17.08 & 3.67 \\
\hline Cohesión & 16 & 50 & 36.75 & 6.78 \\
\hline Adaptabilidad & 13 & 50 & 29.88 & 6.23 \\
\hline Salud física & 8 & 20 & 19.51 & 2.81 \\
\hline Salud mental & 10 & 27 & 15.79 & 4.21 \\
\hline Total salud (CDVRS) & 21 & 47 & 35.29 & 6.30 \\
\hline
\end{tabular}

En dicha tabla se puede observar que el estilo de afrontamiento racional reportó una media elevada y moderada el afrontamiento por desapego emocional, al igual que el estilo de afrontamiento emocional, y finalmente el estilo de afrontamiento por evitación alcanzó una media baja. Se puede evidenciar que los estilos adaptativos fueron los más empleados por la muestra estudiada.

En tanto, los análisis de las dimensiones del funcionamiento familiar, que se reflejan en dicha tabla, indican que la cohesión y la adaptabilidad alcanzaron puntajes moderados. Por último, la calidad de vida relacionada con la salud de los cuidadores se encuentra conservada. En los indicadores que conforman la salud física y la mental, las medias respectivas muestran que la salud física de los cuidadores se encontró más conservada que la salud mental, según los rangos de puntaje en cada una de esas dimensiones.

\section{Análisis de correlación entre variables}

En la Tabla 2 se muestran las correlaciones entre las dimensiones del funcionamiento familiar y el afrontamiento.

Tabla 2. Correlaciones entre las dimensiones del funcionamiento familiar y el afrontamiento.

\begin{tabular}{|l|l|c|c|c|c|}
\cline { 3 - 5 } \multicolumn{2}{c|}{} & $\begin{array}{c}\text { Afrontamiento } \\
\text { emocional }\end{array}$ & $\begin{array}{c}\text { Afrontamiento } \\
\text { racional }\end{array}$ & $\begin{array}{c}\text { Afrontamiento } \\
\text { por desapego }\end{array}$ & $\begin{array}{c}\text { Afrontamiento } \\
\text { evitativo }\end{array}$ \\
\hline \multirow{2}{*}{ Cohesión } & Correlación de Pearson &.$- .336^{* *}$ & $\mathbf{. 2 3 6 * *}$ & -.075 & .079 \\
\cline { 2 - 6 } & Sig. (bilateral) & .000 & .001 & .294 & .265 \\
\hline \multirow{2}{*}{ Adaptabilidad } & Correlación de Pearson & .090 & $\mathbf{. 2 1 5 * *}$ &. $\mathbf{2 1 7 * *}$ & .087 \\
\cline { 2 - 6 } & Sig. (bilateral) & .206 & .002 & .002 & .218 \\
\hline
\end{tabular}

Como se aprecia, la dimensión de cohesión correlacionó de forma inversa y significativa con el estilo de afrontamiento emocional $(p<0.01)$, caso contrario al estilo racional, en el que se reportó una relación positiva, aunque baja y significativa $(p<0.01)$. Esto indica que los cuidadores que perciben una alta cohesión tienden a tener un estilo de afrontamiento racional y no emocional. En los estilos por desapego emocional y evitativo no se hallaron relaciones significativas. La dimensión de adaptabilidad reportó una relación positiva, baja y significativa con el estilo racional y por desapego emocional, reflejando que una percepción de alta adaptabilidad en la familia se relaciona con el empleo de estos estilos adaptativos de afrontamiento.
No se hallaron correlaciones significativas con los estilos desadaptativos.

En la Tabla 3 se reportan las correlaciones obtenidas en las dimensiones de afrontamiento y funcionamiento familiar respecto a la calidad de vida relacionada con la salud de los cuidadores.

Para los estilos de afrontamiento, solo el estilo de afrontamiento emocional correlacionó de forma inversa, aunque baja pero significativa, con la calidad de vida relacionada con la salud $(p<0.05)$ $\mathrm{y}$ los indicadores de salud física $(p<0.05)$ y mental $(p<0.05)$. Con el resto de los estilos de afrontamiento no se hallaron relaciones significativas. Lo mismo se halla con relación a la cohesión y la adaptabilidad familiar, lo que refleja la independencia de estas variables. 
Tabla 3. Correlaciones entre afrontamiento, funcionamiento familiar y calidad de vida relacionada con la salud.

\begin{tabular}{|l|l|c|c|c|}
\cline { 3 - 5 } \multicolumn{2}{c|}{} & Salud física & Salud mental & CDVRS \\
\hline \multirow{2}{*}{ Cohesión } & Correlación de Pearson & .021 & .080 & .061 \\
\cline { 2 - 5 } & Sig. (bilateral) & .773 & .264 & .388 \\
\hline \multirow{2}{*}{ Adaptabilidad } & Correlación de Pearson & -.037 & -.040 & -.049 \\
\cline { 2 - 5 } & Sig. (bilateral) & .600 & .575 & .495 \\
\hline \multirow{2}{*}{$\begin{array}{l}\text { Afrontamiento } \\
\text { por desapego }\end{array}$} & Correlación de Pearson & -.049 & -.113 & -.083 \\
\cline { 2 - 5 } & Sig. (bilateral) & .494 & .111 & .243 \\
\hline $\begin{array}{l}\text { Afrontamiento } \\
\text { racional }\end{array}$ & Correlación de Pearson & .002 & -.128 & -.056 \\
\cline { 2 - 5 } & Sig. (bilateral) & .980 & .071 & .432 \\
\hline \multirow{2}{*}{$\begin{array}{l}\text { Afrontamiento } \\
\text { emocional }\end{array}$} & Correlación de Pearson & $-.142^{*}$ & $-.155^{*}$ & $-.164^{*}$ \\
\cline { 2 - 5 } & Sig. (bilateral) & .044 & .028 & .020 \\
\hline $\begin{array}{l}\text { Afrontamiento } \\
\text { evitativo }\end{array}$ & Correlación de Pearson & .009 & -.072 & -.027 \\
\cline { 2 - 5 } & Sig. (bilateral) & .904 & .308 & .709 \\
\hline
\end{tabular}

\section{DISCUSION}

El presente estudio tuvo como objetivo determinar la correlación entre los estilos de afrontamiento, el funcionamiento familiar y la calidad de vida relacionada con la salud en una muestra de cuidadores de pacientes oncológicos venezolanos.

En dicho estudio, la mayoría de los participantes fueron mujeres, adultas jóvenes y de estratos socioeconómicos distintos, lo que confirma lo hallado en un estudio previo de Carratalá y Peña (2009) sobre el perfil del cuidador del paciente oncológico venezolano, que se caracteriza por ser los cuidadores frecuentemente mujeres. Especialmente en los países latinos se sostiene la creencia de que las actividades de cuidar se hallan asociadas al sexo femenino, indistintamente del parentesco que tengan con el paciente diagnosticado (Dakduk, 2010). Otro aspecto importante se relaciona con el número de horas diarias dedicadas al cuidado del familiar diagnosticado que reportan los cuidadores, que en esta muestra fue de diecisiete horas diarias de cuidado en promedio. En el estudio de Carratalá y Peña (2009) se encontró que los cuidadores de pacientes oncológicos cuidaban, en promedio, siete horas. Este dato llama la atención porque, aunque los cuidadores de la muestra hayan sido mayormente adultos jóvenes y en las condiciones físicas necesarias para realizar las labores de cuidado, se hallaban en riesgo de sufrir una sobrecarga por el número de horas dedicadas a esa actividad. Algunos autores (Pérez et al., 2016; Williams, 2018) han señalado que las horas de cuidado pueden incidir en la organización del tiempo y de forma negativa en la salud de los cuidadores. Pérez, Rascón y Villafaña (2014) hallaron que cuando la salud mental del cuidador es baja, disminuye el afrontamiento activo debido, en parte, al aumento en el número de horas que dedica al cuidado.

El análisis de las variables muestra que los cuidadores de los pacientes oncológicos tienden en mayor medida a emplear los estilos de afrontamiento racional y por despego emocional. De este modo, los cuidadores tienden a afrontar el estresor de la enfermedad a través de resolver los problemas, además de evaluarlos objetivamente sin implicar en ello sus emociones (Roger et al., 1993). Lazarus y Folkman (1986) han señalado que el afrontamiento orientado al problema promueve la adaptación favorable de los individuos a los estresores que perciben como amenazantes para su bienestar. Tales resultados son consistentes con los de otros estudios (Flórez y Klimenko, 2011; Pérez et al., 2016; Trask et al., 2003) que han reportado que el uso de estrategias de afrontamiento adaptativas por parte de los cuidadores de pacientes oncológicos les permite reducir su estrés psicológico.

Se encontró el empleo del afrontamiento emocional, aunque de forma moderada y en menor medida el evitativo. Es importante señalar que, ante el diagnóstico de cáncer en un familiar, es de esperarse que este tipo de afrontamiento ocurra en 
virtud del impacto emocional que representa para los miembros de la familia ese evento (Williams, 2018). El estigma asociado al cáncer como sinónimo de muerte y sufrimiento suscitan reacciones emocionales de incertidumbre, desesperanza, angustia y temor que pueden afectar el funcionamiento del paciente y el de su familia (Alderfer y Kazak, 2006; Garassini, 2016, Olivares, 2015). De igual manera, la menor tendencia hallada al afrontamiento evitativo sugiere que los cuidadores de la muestra no evitaban la situación estresante mientras ocurría. El cáncer es un suceso en el que los cuidadores, al asumir conscientemente ese rol, deben mantenerse activos en todas las tareas instrumentales y emocionales que supone el proceso del cuidar (Sánchez y Gasperi, 2012), y por ello es de esperarse que no recurran a evitar la situación.

Por otra parte, los cuidadores percibieron en su familia una alta cohesión (Olson, 2000, Olson et al., 2019) esto es, una familia unida en una situación de gran estrés, lo que respalda la idea de que la cohesión entre los miembros de la familia puede ser un factor protector para afrontar las adversidades, como la enfermedad de uno de sus miembros (Garassini, 2016; Walsh, 2016). Respecto a la adaptabilidad familiar, se obtuvo un puntaje elevado, lo que, según el modelo propuesto por Olson et al. (2019), indica una baja adaptación, lo que sugiere que no se percibe la familia como capaz de cambiar sus roles y las reglas en sus relaciones cuando atraviesan por el estresor que significa la enfermedad oncológica en uno de sus miembros.

Este resultado cobra sentido cuando se percibe que el cuidador principal es el único que asume todas las tareas del cuidado, sin delegar funciones a otros miembros, lo que podría aumentar los conflictos entre ellos e influir en la salud del propio cuidador (Carratalá y Peña, 2009; Garassini, 2015, 2016). En el modelo circumplejo se considera la comunicación como una dimensión que se interconecta con la cohesión y la adaptabilidad. Para efecto de los resultados, podría pensarse que los cuidadores carecen de estrategias para comunicarse adecuadamente mientras está presente el estresor, lo que conlleva la percepción de un funcionamiento familiar poco favorable. La comunicación es un elemento clave que permite que las familias puedan organizarse, resolver problemas y apoyarse ante eventos estresantes, como la enfermedad de cáncer en un familiar (Alderfer, Navsaria y Kazak, 2009; Adelfer y Stanley, 2012; Baider, 2003; Walsh, 2016). En el estudio de Lim y Shon (2018) se encontró que la comunicación era un factor que mediaba la relación entre la cohesión y la calidad de vida relacionada con la salud de cuidadores esposos de mujeres sobrevivientes.

Se evidenció que la calidad de vida relacionada con la salud global de los cuidadores estaba conservada en la muestra. Específicamente, la salud física de los cuidadores mostró estar menos deteriorada que su salud mental, la que se ubicó en niveles moderados. Estos resultados pueden explicarse por las características de la muestra, constituida sobre todo por adultos jóvenes, quienes por lo general son los que se encuentran en las condiciones físicas apropiadas para asumir las tareas del cuidado. Los niveles de salud mental pudieran explicarse por la vivencia del impacto emocional que trae consigo cuidar a un paciente oncológico. Se sabe que muchos cuidadores en el proceso del cuidar pueden experimentar sintomatología asociada al estrés crónico, como ansiedad, depresión, aislamiento social y conductas de riesgo tales como consumir en exceso alcohol y tabaco (Tripodo, Veloso y Llanos, 2015; Williams, 2018).

En el análisis de las correlaciones se obtuvieron relaciones significativas entre algunos estilos de afrontamiento y la cohesión y adaptabilidad familiares. Se encontró que los cuidadores que perciben a su familia como cohesionada tienden a tener un estilo de afrontamiento racional $y$, en menor medida, emocional. Por otra parte, aquellos que perciben a su familia como flexible tienden a tener un estilo de afrontamiento racional y por desapego emocional ante los estresores de la enfermedad. Estos resultados son consistentes con evidencias previas (Acosta et al., 2017; Cortés et al., 2012; Díaz y Yaringaño, 2010) en las que se encontró que un adecuado clima y un buen funcionamiento familiar permite el empleo de estrategias apropiadas de afrontamiento, lo que implica experimentar menos estrés y, por ende, a mantener una mejor salud en los cuidadores y en los pacientes que atienden.

Finalmente, no se hallaron correlaciones significativas entre los estilos de afrontamiento y la 
calidad de vida relacionada con la salud, excepto en el afrontamiento emocional, correlación que fue inversa y significativa, lo que implica que la tendencia a emplear este último se relaciona con el deterioro de la salud de los cuidadores. La cohesión y la adaptabilidad no correlacionaron con ninguno de los indicadores de la calidad de vida relacionada con la salud, lo que sugiere que, independientemente de que la muestra de cuidadores de pacientes oncológicos percibiera como favorable o no el funcionamiento de su familia, o de que mantuviera o no estilos de afrontamiento adaptativos, al parecer no tuvo implicaciones en dicha calidad de vida relacionada con la salud.

La correlación obtenida entre los estilos de afrontamiento adaptativos no es consistente con los resultados de investigaciones previas (Aldana y Guarino, 2012; Torres y Guarino, 2013; Sojo et al., 2007), en las que ha reportado que ambos estilos de afrontamiento se asocian con un aumento de la salud global, mientras que la relación con el estilo emocional encontró respaldo en los estudios antes referidos. Esto último apoya la idea de que el estilo de afrontamiento emocional puede generar un deterioro de la salud siempre que sea prolongado (Lazarus y Folkman, 1986).

Sobre las correlaciones obtenidas con los estilos adaptativos, podría suponerse la existencia de otro estilo de afrontamiento que tenga más influencia en la salud de los cuidadores que el estilo racional o el de desapego emocional, o bien que intervengan ciertas características de la personalidad de los propios cuidadores. Es posible que esta forma de manejo esté referida a un estilo de afrontamiento represivo, caracterizado por evitar pensamientos, emociones y recuerdos desagradables de los eventos estresores, lo que podría deteriorar la salud en el largo plazo (Weinberger, 1979). Sin embargo, algunos estudios (Bonnano, 2008: Caro y Cova, 2012; Ginzburg, Solomon y Bleich, 2002; Parker y McNally, 2008) han encontrado que ese estilo de afrontamiento no necesariamente resulta ser perjudicial para la salud si persiste. Parker y McNally (2008) han señalado que lo anterior pudiera estar mediado por la existencia de un sesgo interpretativo de los represores, vinculado con la deseabilidad social, que haría que los cuidadores atribuyeran un significado más positivo a sus pensamientos y emociones. Así, ante el impacto emo- cional del diagnóstico, los cuidadores, empleando el afrontamiento represivo, podrían no experimentar sus emociones desagradables, disociándolas, y seguir cumpliendo con las tareas de cuidado al verlas como una forma adaptativa de afrontamiento, al igual que los estilos racional y por desapego emocional. No obstante, tal suposición debe ser corroborada en futuros estudios que determinen las relaciones entre los estilos de afrontamiento propuestos por Roger et al. (1993) y el estilo de afrontamiento represivo, así como la influencia de este último en la calidad de vida relacionada con la salud de los cuidadores de pacientes oncológicos. De igual forma, no se puede descartar la influencia de otras variables, por ejemplo el número de horas de cuidado, que resultó ser bastante elevado, lo que puede implicar un deterioro en el funcionamiento global del cuidador y afectar el uso de estrategias de afrontamiento eficaces (Pérez et al., 2014; Tsai y Jirovec, 2005).

De hecho, las características de personalidad pueden influir en la salud de los cuidadores. Las características de personalidad resistente (Kobasa, Maddi y Puccetti, 1982), por ejemplo, suponen que existen ciertos rasgos en los individuos que hacen que sus experiencias estresantes no tengan un efecto negativo. Se ha encontrado que tener una personalidad resistente puede llevar a emplear mejores estrategias de afrontamiento, disminuyendo así los efectos negativos del estrés e incrementando, por ende, la salud y el bienestar (Moreno, Garrosa, Corso, Boada y Rodríguez, 2012). Donoso y Barra (2013) encontraron que la personalidad resistente en cien cuidadoras de pacientes con cáncer de mama se asoció a un menor estrés percibido y al aumento de su bienestar psicológico.

Aun así, es importante que se aporten más evidencias que corroboren la consistencia de las correlaciones reportadas en el presente estudio, subrayando la relación entre el funcionamiento familiar y la calidad relacionada con la salud de los cuidadores, dada la importancia que este tipo de apoyo tiene para ellos. El estudio provee una aportación al estudio de los factores psicosociales que pueden influir en el mantenimiento de la salud de los cuidadores de pacientes oncológicos adultos, área que ha sido mayormente abordada con cuidadores de pacientes oncológicos pediátricos (Cortés et al., 2012; Van Schoors et al., 2017). 
La relevancia social de este estudio se focaliza en la importancia de promover de forma continua una atención psicooncológica grupal, familiar e individual a los cuidadores de los pacientes, pues este tipo de apoyo puede proporcionarles una oportunidad para que adquieran y ejerzan estrategias de afrontamiento que les haga posible llevar a cabo las demandas de esa tarea. Se rescata aquí la importancia de que en las intervenciones parti- cipen los demás miembros de la familia para que conozcan las labores que implica el cuidado del familiar enfermo, toda vez que eso le permite al cuidador principal afrontar el estrés excesivo que implica cuidar, lo que puede lograrse estimulándolos mediante una comunicación adecuada que les facilite su ajuste a las diferentes demandas de la enfermedad, logrando así un mejor funcionamiento ante tal estresor.

\section{REFERENCIAS}

Acosta, E., López, C., Martínez, M. y Zapata, R. (2017). Funcionalidad familiar y estrategias de afrontamiento en pacientes con cáncer de mama. Horizonte Sanitario, 16(2), 139-148.

Aldana, G. y Guarino, L. (2012). Sobrecarga, afrontamiento y salud en cuidadoras de pacientes con demencia tipo alzheimer. Summa Psicológica UST, 9(1) 5-14.

Alderfer, M. y Kazak, A. (2006). Family issues when a child is on treatment for cancer. En R. Brown (Ed.): Pediatric Hematology/ Oncology: Biopsychosocial approach (pp. 53-74). New York: Oxford University Press.

Alderfer, M., Navsaria, N. y Kazak, A. (2009). Family functioning and posttraumatic stress disorder in adolescent survivors of childhood cancer. Journal of Family Psychology, 23(5), 717-725.

Alderfer, M. y Stanley, C. (2012). Health and illness in the context of the family. En A. Baum, A. Revenson y J. Singer (Eds.): Handbook of Health Psychology (2a ed.) (pp. 493-516). London: Taylor \& Francis.

Ariza, E., Barrera, M. y Pinto, T. (2009). Afrontamiento ante el diagnóstico de cáncer. Revista de Psicología GEPU, 1(2), 19-29.

Baider, L. (2003). Cáncer y familia: aspectos teóricos y terapéuticos. Revista Internacional de Psicología Clínica y de la Salud, 3(3), 505-520.

Bayen, E., Laigle-Donadey, F. y Proute, M. (2016). The multidimensional burden of informal caregivers in primary malignant brain tumor. Support Care Cancer, 25(1), 245-253.

Bazo, J., Bazo, O., Águila, J., Peralta, F., Mormontoy, W. y Bennett, I. (2016). Propiedades psicométricas de la Escala de Funcionalidad Familiar FACES III: un estudio en adolescentes peruanos. Revista Peruana de Medicina Experimental y Salud Pública, 33(1), 462-470.

Biffi, R. y Mamede, M. (2010). Perception of family functioning among relatives of women who survived breast cancer: gender differences. Revista Latinoamericana de Enfermagem, 18(2), 269-277.

Bonnano, G. (2008). Loss, trauma, and human resilience: have we underestimated the human capacity to thrive after extremely aversive events? Psychological Trauma: Theory, Research, Practice, and Policy, 8(1), 101-113.

Caro, P. y Cova, F. (2012). Relacion entre estrés postraumatico, afrontamiento represivo y rumiacion en estudiantes univerisitarios chilenos. Ciencia y Enfermeria, 13(3), 121- 130.

Carratalá, A. y Peña, C. (2009). Resiliencia en familiares acompañantes de pacientes oncológicos. Tesis inédita de pregrado. Caracas: Universidad Metropolitana.

Cortés, F., Bueno, J., Narváez, A., García, A. y Guerrero, L. (2012). Funcionamiento familiar y adaptación psicológica en oncología. Psicooncología, 9(2-3), 335-354.

Dakduk, S. (2010). Enjevececer en casa: el rol de la mujer como cuidadora de familiares mayores dependientes. Revista Venezolana de Estudios de la Mujer, 15(35), 79-90.

Díaz, G. y Yaringaño, J. (2010). Clima familiar y afrontamiento al estrés en pacientes oncológicos. IIPSI, 13(1), 69-86.

Donoso, V. y Barra, E. (2013). Personalidad resistente, estrés percibido y bienestar psicológico en cuidadoras familiares de pacientes con cáncer terminal. Psicologia y Salud, 23(2), 153-160.

Expósito, Y. (2008). La calidad de vida en los cuidadores primarios de pacientes con cáncer. Revista Habanera de Ciencias Médicas, 7(3). Recuperado de http://scielo.sld.cu/scielo.php?script=sci_arttextypid=S1729-519X2008000300004.

Fernández, J. Fernández, M. y Cieza, A. (2010). Los conceptos de calidad de vida, salud, y bienestar analizados desde la perspectiva de la Clasificación Internacional de Funcionamiento (CIF). Revista Española de Salud Pública, 84(2), $169-184$.

Flórez, J. y Klimenko, O. (2011). Estrategias de afrontamiento en pacientes oncológicos y sus cuidadores, inscritos al Programa de Asistencia Paliativa de la Corporación FORMAR. (Tesis inédita de pregrado). Medellín (Colombia): Universidad de Envigado. 
Fuchs-Tarlovsky, V., Bejarano, M., Álvarez A., Godoy, M. y Fernández, K. (2013). Efecto de la presencia de los familiares sobre la depresión en mujeres hospitalizadas con cáncer. Revista Venezolana de Oncología, 25(2) 190-195.

Garassini, M. (2015). Narrativas de familiares de pacientes con cáncer. Revista CES Psicología, 8(2), 76-102.

Garassini, M. (2016). Los cuidadores de pacientes con cáncer: aprendizajes y recomendaciones a las familias, sociedades y centros de salud. Psicología y Salud, 26(2), 161-175.

García, B. (2011). Estudio cualitativo del perfil del cuidador primario, sus estilos de afrontamiento y el vínculo afectivo con el enfermo oncológico infantil. Tesis doctoral inédita. Barcelona: Universitat Ramon Llul.

Ginzburg, K., Solomon, Z. y Bleich, A. (2002). Repressive coping style, cute stress disorder, and posttraumatic stress disorder after myocardial infarction. Psychosomatic Medicine, 64, 748-757.

González, A., Fonseca, M., Valladares, A. y López, L. (2017). Factores moduladores de resiliencia y sobrecarga en cuidadores principales de pacientes oncológicos avanzados. Revista Finlay, 5(2), 26-32.

Hanly, P., Maguire, R., Balfe, M., Hyland, P., Timmons, A., O'Sullivan, E., Butow, P. y Sharp, L. (2016). Burden and happiness in head and neck cancer carers: the role of supportive care needs. Support Care Cancer, 24, 4283-4291.

Hernández, R., Fernández, C. y Baptista, L. (2012). Metodología de la investigación. México: McGraw Hill.

Holland, J., Breitbart, W., Butow, P., Jacobsen, P., Loscalzo, M. y McCorkle, R. (2015). Psycho-Oncology (3a ed). New York: Oxford University Press.

Jung, J., Lee, J., Kim, M., Shim, Y., Zo, J. y Yun, Y. (2017). Comparison of fatigue, depression, and anxiety as factors affecting posttreatment health-related quality of life in lung cancer survivors. Journal of Psychology, 16(4), 167-146.

Karimi, M. y Braizer, J. (2016). Health, health-related quality of life, and quality of life: what is the difference? Pharmacoeconomics, 34(7), 645-649.

Kelinger, F. y Lee, H. (2002). Investigación del comportamiento. Buenos Aires: McGraw-Hill.

Kissane, D. y Parne, F. (2015). Bereavement care for families. New York: Routledge.

Kobasa, S., Maddi, M.S y Puccetti, C. (1982). Personality and exercise as buffers in the stress-illness relationship. Journal of Behavioral Medicine, 5(4), 391-404.

Lambert, S., Levesque, J. y Girgis, A. (2016). The impact of cancer and chronic conditions on caregirvers and family members. En B. Koczwara (Ed.): Cancer and chronic conditions. Singapore: Springer.

Lazarus, R. y Folkman, S. (1986). Estrés y procesos cognitivos. Madrid: Martínez-Roca.

Leroy, T., Fournier, E., Penel, N. y Christophe, V. (2016). Crossed views of burden and emotional distress of cancer patients and family caregivers during palliative care. Psycho-Oncology, 25, 1278-1285.

Lim, J. y Shon, E. (2018). The dyadic effects of family cohesion and comunication on health-related quality of life. Cancer Nursing, 42(2), 156-165.

Martin, S., Calabrese, S., Wolters, P., Walker, K., Warren, K. y Hazra, R. (2012). Family functioninig and coping styles in families of children with cancer and HIV disease. Clinical Pediatrics, 51(1), 58-64.

Moreno, B., Garrosa, E., Corso, S., Boada, M. y Rodríguez, R. (2012). Personalidad resistente y capital psicológico: Las variables personales positivas y los procesos de agotamiento y vigor. Psicothema, 24(1), 79-86.

Olivares, M. (2015). Trastorno de estrés postraumático asociado al cáncer. Revisión teórica. Ansiedad y Estrés, 2(6), 79-87.

Olson, D.H. (2000). Circumplex model of marital and family systems. Journal of Family Therapy, 22, 144-167.

Olson, D.H., Portner, J. y Bell, R.Q. (1982). Faces II: Family Adaptability and Cohesion Evaluation Scales. Minneapolis, MI: University Of Minnesota.

Olson, D.H., Waldvogel, L. y Schlieff, M. (2019). Circumplex Model of marital and family systems: An update. Journal of Family: Theory \& Review, 11(2), 199-211.

Organización Mundial de la Salud (2018). Documentos Básicos. Ginebra: OMS.

Parker, H. y McNally, R. (2008). Repressive coping, emotional adjustment, and cognition in people who have lost loved ones to suicide. Suicide and Life-Threatening Behavior, 38(6), 676-687.

Pérez O., F., Frías O., A., Romero, R. y Del Pino C., R. (2016). Coping strategies and anxiety in caregivers of palliative cancer patients. European Journal of Cancer Care, 25(4), 600-607.

Pérez, I., Rascón, M. y Villafaña, J. (2014). Coping and emotional discomfort in primary caregivers of cancer patients. Psycho-Oncology, 27(2), 120-134.

Ramírez, S. (2017). Funcionalidad familiar y su asociación con la sobrecarga del cuidador primario en pacientes con cáncer de mama de la UMF 75 Nezahualcóyotl. (Tesis inédita de maestría). Toluca (México): Universidad Autónoma del Estado de México.

Ramírez, R., Agredo, R. y Jerez, A. (2010). Confiabilidad y valores normativos preliminares del cuestionario de la salud SF-12 (Short Form 12 Health Survey) en adultos colombianos. Revista de Salud Pública, 12(5), 807-819. 
Roger, D., Jarvis, G. y Najarian, B. (1993). Detachment and coping: the construction and validation of a new scale for measuring coping strategies. Personality and Individual Differences, 15(6), 61-66.

Rogero, J. (2010). Las consecuencias del cuidado familiar sobre el cuidador: una valoración compleja y necesaria. Index de Enfermería, 19(1), 47-50.

Rosenstiel, A.K. y Keefe, F.J. (1983). The Coping Style Questionnaire (CSQ). Pain, 17, 33-44.

Rumpold, T., Schur, S., Amering, M., Kirchheiner, K., Masel, E.K., Watzke, H. y Schrank, B. (2016). Informal caregivers of advanced-stage cancer patients: every second is at risk for psychiatric morbidity. Support Care Cancer, 24(5), 1975-1982.

Sánchez, Y. y Gasperi, R. (2012). Perfil del cuidador informal y nivel de conocimientos sobre cuidados paliativos domiciliario en estado terminal. Salud, Arte y Cuidado, 5(2), 4-16.

Sojo, V., Bethelmy, L. y Guarino, L. (2007). Adaptación y validación preliminar de la versión hispana del Cuestionario de Estilos de Afrontamiento. Psicología Conductual, 15(2), 173-189.

Tokem, Y., Hanife, O. y Atiye, C. (2015). Examination of the relationship between hopelessness levels and coping strategies among the family caregirvers of patients with cancer. Cancer Nursing, 18(4), 28-34.

Torres, B. y Guarino, L. (2013). Diferencias individuales y burnout en médicos oncólogos venezolanos. Universitas Psychologica, 12(1), 95-104.

Trask, P., Paterson, A., Trask, C., Bares, C., Birt, J. y Maan, C. (2003). Parent and adolescent adjustment to pediatric cancer: Associations with coping, social support and family function. Journal of Pediatric Oncology Nursing, 20(1), 36-47.

Tripodo, V., Veloso, V., L. y Llanos, V. (2015). Sobrecarga del cuidador principal de pacientes en cuidados paliativos. Argumentos, 17, 307-330.

Tsai, P. y Jirovec, M. (2005). The relationships between depression and other outcomes of chronic illness caregiving. BMC Nursing, 4(3), 1-10.

Urzúa, A. (2010). Calidad de vida relacionada con la salud: elementos conceptuales. Revista Médica de Chile, 138(3), 358-365.

Van Schoors, M., Caes, L., Knoble, N., Goubert, L., Verhofstadt, L. y Alderfer, M. (2017). Systematic review: associations between family functioning and child adjustment after pediatric cancer diagnosis: A meta-analysis. Journal of Pediatric Psychology, 42(1), 6-18.

Van Schoors, M., Caes, L., Verhofstadt, L., Goubert, L. y Alderfer, M. (2015). Systematic review: family resilience after pediatric cancer diagnosis. Journal of Pediatric Psychology, 40(9), 856-868.

Velázquez, A., García, M., Alvarado, A. y Sánchez, A. (2007). Psicología y cáncer. Gaceta Mexicana de Oncología, 6(3), 71-75.

Walsh, F. (2016). Strengthening family resilience (3a ed.). New York: The Guilford Press.

Ware, J.E. Jr., Kosinski, M. y Keller, S.D. (1996). A 12-Item Short-Form Health Survey: construction of scales and preliminary tests of reliability and validity. Med Care, 34, 220-233.

Watson, M. y Greer, S. (1998). Development of a questionnaire for the measure of adjustment to cancer: The MAC scale. Psychological Medicine, 18(5), 203-209.

Weinberger, D. (1979). Low-anxious, high-anxious, and repressive coping styles: Psychometric patterns and behavioral and physiological responses to stress. Journal of Abnormal Psychology, 88(4), 369-380.

Williams, A. (2018). Family caregivers to adults with cancer: The consequences of caring. Psycho-Oncology. Recent Results in Cancer Research, 210, 87-103. doi: 10.1007/978-3-319-64310-6_6. 